\title{
The Microbiome in Psoriasis and Psoriatic Arthritis: The Skin Perspective
}

\author{
Hok Bing Thio
}

\begin{abstract}
Psoriasis is a chronic, inflammatory immune-mediated skin disease that affects about $2 \%$ of the world's population. In $20 \%$ of patients with psoriasis, the characteristic skin lesions are accompanied by psoriatic arthritis (PsA). Psoriasis arises in genetically predisposed individuals who have a dysregulated immune response to various environmental factors. The human body is home to many microbial species, and both the skin and the gut microbiome influence the development and function of immune tissue development and function. Studies on the cutaneous microbiome show a trend toward an increased relative abundance of Streptococcus and a decreased level of Propionibacterium in patients with psoriasis compared to healthy controls. In the gut microbiome, the ratio of Firmicutes and Bacteroidetes was perturbed in psoriatic individuals compared to healthy controls. Actinobacteria was relatively underrepresented in patients with psoriasis compared to healthy individuals. A decrease in skin microbiome flora diversity seems to be a sign that a patient with psoriasis is at elevated risk for developing arthritis. Modulating the skin microbiota for therapeutic reasons can be achieved by antimicrobial (antibiotic) therapy, the application of prebiotics or probiotics, or the transplantation of an entire healthy microbial population. (J Rheumatol Suppl. 2018 June;94:30-1; doi:10.3899/ jrheum.180133)
\end{abstract}

Key Indexing Terms:

MICROBIOME SKIN GRAPPA

Psoriasis is an inflammatory, immune-mediated skin disease that affects about $2 \%$ of the world's population. In $20 \%$ of patients with psoriasis, red, scaling skin lesions are accompanied by psoriatic arthritis (PsA). The skin harbors a wide range of different cell populations, including keratinocytes, macrophages, dendritic cells, innate lymphocytes, and different $\mathrm{T}$ cell populations that belong to or perform functions of the innate and adaptive immune system. Psoriasis arises in genetically predisposed individuals who have a dysregulated immune response to various environmental factors. Similar to the gastrointestinal (GI) tract, the skin is a dynamic living interface between an individual and the exogenous environment. Studies have identified an array of genetic, epigenetic, and immunological factors that are involved in the disease pathogenesis of psoriasis and PsA. The human body is home to multiple microbial species (bacteria, fungi, parasites, and viruses), and the genetic materials of all these microorganisms form the microbiome. The microbiome was not generally recognized to exist until

From the Department of Dermatology, Erasmus University Medical Centre, Rotterdam, the Netherlands.

As part of the supplement series GRAPPA 2017, this report was reviewed internally and approved by the Guest Editors for integrity, accuracy, and consistency with scientific and ethical standards.

H.B. Thio, MD, PhD, Department of Dermatology, Erasmus University Medical Centre.

Address correspondence to Dr. H.B. Thio, Department of Dermatology, Erasmus University Medical Centre (room GK-218), Burgemeester s'Jacobplein 51, 3015CA Rotterdam, P.O. Box 2040, 3000CA Rotterdam, the Netherlands.E-mail:h.thio@erasmusmc.nl

the late 1990s. Both the skin and the gut microbiome influence the development and function of immune tissue.

The microbiome has been implicated as a potential trigger for immune dysregulation, which promotes systemic inflammation ${ }^{1}$. Microbial profiling has revealed the presence of highly diverse commensal communities along distinct topographical skin sites. While a healthy skin microbiome functions to protect its host, an increased or decreased skin microbiome bacterial composition (called dysbiosis) leads to skin inflammation and disease. Assuming a skin surface of $2 \mathrm{~m}^{2}$ and maximum microbial (bacterial) cell densities of about $10^{6}$ per $\mathrm{cm}^{2}$, the skin of healthy adults can be estimated to be colonized by a maximum of $10^{10}$ microorganisms in total. However, bacteria are distributed very unevenly across the different niches of the human skin. Cell numbers range from about $10^{2}$ per $\mathrm{cm}^{2}$ (fingertips, back) to $10^{6}$ per $\mathrm{cm}^{2}$ (forehead, axilla). Together, Firmicutes, Actinobacteria, Bacteroidetes, and Proteobacteria form the largest microbial populations that inhabit the skin. Human skin microbiota is heterogeneous because of the existence of several microhabitats that are characterized by the predominance of a specific bacteria: sebaceous sites with Propionibacterium spp, moist sites (such as axilla and inguinal crease) with Staphylococcus and Corynebacterium spp, and dry sites with gram-negative microorganisms. While it was long believed that microbial life on healthy skin was restricted to epidermis, hair follicles, and sebaceous and sweat glands, various analyses suggest that viable microbes are also found in deeper skin layers, i.e., the dermis and underlying fat tissue. In humans, the microbial

Personal non-commercial use only. The Journal of Rheumatology Copyright $($ C 2018. All rights reserved. 
variation is higher among different microhabitat skin sites of the same individual than among skin sites from the same microhabitat of different individuals.

Although the psoriatic microbiome field is relatively new, initial studies on the cutaneous microbial compositions of subjects with psoriasis and PsA in both psoriatic lesions and unaffected skin reveal interesting differences in microbiome composition $^{2,3}$. Studies on the cutaneous microbiome show a trend toward an increased relative abundance of Streptococcus and a decreased level of Propionibacterium in patients with psoriasis compared to healthy controls. In the gut microbiome, the ratio of Firmicutes and Bacteroidetes has been shown to be perturbed in individuals with psoriatic disease compared to healthy controls ${ }^{2}$. Actinobacteria has also been shown to be relatively underrepresented in patients with psoriasis compared to healthy individuals ${ }^{2}$. Staphylococcus and Corynebacterium have been the predominant bacteria in all samples. It seems that a decrease in skin microbiome flora diversity could be an initial sign that a patient with psoriasis is at an elevated risk for developing Ps $A^{4}$.

Given the interindividual variation in the composition of the (skin) microbiota of healthy subjects, it is challenging to define a standard "healthy" skin; however, it is generally believed that a healthy skin microbiota is characterized by a high diversity of commensal or even beneficial (symbiotic) bacteria. In contrast, disease (in our case psoriasis) is thought to be associated with a dysbalanced microbiota (dysbiosis) characterized by a loss of microbial diversity and an increase in absolute numbers and relative abundance of pathogenic bacteria.

Next to the role of specific gut bacteria, the human fungal and yeast microbial community (mycobiome) has been appreciated as important for health ${ }^{5}$. The majority of a human host's interactions with yeast and fungi take place in the intestines and skin. Recent studies identified hundreds of different types of fungi in oral and colonic microbiota. Most fungi are resident to skin, genital, and GI mucosa without causing disease.

An interesting report in this field is that the baker's yeast, Saccharomyces cerevisiae, a highly abundant fungus in the human gut, has been found to be decreased in the gut of patients with psoriasis, but appears to be restored upon oral immunomodulatory therapy with dimethylfumarate ${ }^{6}$. $S$. cerevisiae is generally classified as a yeast with beneficial immunomodulatory properties. In psoriasis, there are many therapeutic options, including topical treatment, ultraviolet therapy, conventional systemic therapy such as dimethylfumarate and cyclosporin, and biologic therapy. The effects of these therapies on the GI and cutaneous microbiome and mycobiome still need to be elucidated.
Different factors, such as the environment, host genetic variation, lifestyle, and hygiene may cause shifts in the microbiota of the skin. These shifts in microbiota structure and composition could establish a dysbiotic state that, if not recovered, could result in a dermatologic immune dysregulation state such as psoriasis. The accessibility of skin coupled with the longitudinal stability of skin microbiota allow clinical studies to investigate alterations at different stages of disease states in psoriasis. In addition, rapid and high throughput DNA sequencing platforms and data integration can shed light on the individualized skin microbiome for personalized skin care and therapeutics. Further, patients with psoriasis have a higher prevalence of associated comorbid diseases, including cardiometabolic dysfunction, depression, and metabolic syndrome. Whether the gut and skin microbiota are involved in this comorbidity association is unclear.

Manipulating the skin microbiota for therapeutic reasons can be achieved by antimicrobial (antibiotic) therapy, the application of prebiotics or probiotics, or the transplantation of an entire microbial population to diseased skin. While antibiotics aim to eliminate specific (pathogenic) microorganisms, the main purpose of applying probiotics or transplanting microbiota is to increase selected, beneficial microorganisms.

Microbiome research is currently very popular. Many associations with health and disease outcomes are being investigated, including associations with psoriasis and PsA, and several types of microbiota-related interventions are being developed.

\section{REFERENCES}

1. Egert M, Simmering R, Riedel CU. The association of the skin microbiota with health, immunity, and disease. Clin Pharmacol Ther 2017 Apr 5 (E-pub ahead of print).

2. Yan D, Issa N, Afifi L, Jeon C, Chang HW, Liao W. The role of the skin and gut microbiome in psoriatic disease. Curr Dermatol Rep 2017;6:94-103.

3. Tett A, Pasolli E, Farina S, Truong DT, Asnicar F, Zolfo M, et al. Unexplored diversity and strain-level structure of the skin microbiome associated with psoriasis. NPJ Biofilms Microbiomes 2017;3:14.

4. Langan EA, Griffiths CEM, Solbach W, Knobloch JK, Zillikens D, Thaci D. The role of the microbiome in psoriasis: moving from disease description to treatment prediction? Br J Dermatol 2017 Oct 26 (E-pub ahead of print).

5. Nakajima S, Harrison O, Linehan JL, Belkaid Y. Candida albicans colonization exacerbates skin inflammation in a murine model of psoriasis. J Immunol 2017;198 Suppl:149-16.

6. Eppinga H, Thio HB, Schreurs MWJ, Blakaj B, Tahitu RI, Konstantinov SR, et al. Depletion of Saccharomyces cerevisiae in psoriasis patients, restored by Dimethylfumarate therapy (DMF). PLoS One 2017;12:e0176955. 